\title{
Farmacología y Toxicología
}

\author{
Actividad ixodicida de Phytolacca bogotensis K. y Urtica dioca \\ L. sobre Rhipicephalus (Boophilus) microplus \\ Ixodicide activity of Phytolacca bogotensis $K$. and Urtica dioica $L$. \\ on Rhipicephalus (Boophilus) microplus \\ Carlos E Rodríguez Molano, Zoot, MSc; Néstor J Pulido Suarez, MVZ, MSc(c); \\ Alison J Camargo, Est MVZ. \\ Grupo de Investigación en Bioquímica y Nutrición Animal, Universidad \\ Pedagógica y Tecnológica de Colombia, Sogamoso, Colombia. \\ E-mail:nestor.pulido@uptc.edu.co
}

Introducción: Las garrapatas y las enfermedades que transmiten son una de las principales causas de pérdidas económicas a la ganadería bovina. La forma de controlar esta plaga ha sido a base de ixodicidas, pero debido al uso irracional se ha generado resistencia a estos químicos. Esto ha llevado a la búsqueda de alternativas para el control de esta plaga, como el uso de extractos de plantas. Objetivo: Evaluar la actividad ixodicida in vitro del extracto de Phytolacca bogotensis K. y Urtica dioca L. sobre Rhipicephalus (Boophilus) microplus. Metodos: Los extractos de las plantas se obtuvieron por el método de extracción en caliente (Soxhlet), utilizando únicamente las hojas. Los extractos se sometieron a pruebas cualitativas para determinar los posibles metabolitos presentes en cada uno de ellos. Para los ensayos se utilizaron garrapatas adultas que fueron expuestas a cada uno de los extractos por medio de la prueba de inmersión de adultas. La eficacia de la mortalidad se hizo a las 24, 48, 72 y $96 \mathrm{~h}$ post-exposición, teniendo en cuenta como mínimo eficaz del $60 \%$ de mortalidad. Inicialmente se usó el extracto puro y si presentaba eficacia se procedía a realizar diluciones hasta encontrar la concentración mínima eficaz. Resultados: Los resultados fueron de 87,5, 60 y $25 \%$ de mortalidad usando la hoja de la Phytolacca bogotensis K. con el extracto puro, dilución $1: 2$ y dilución 1:4, respectivamente. Se observó una mortalidad del $25 \%$ con Urtica dioica L., únicamente con el extracto puro. Conclusión: Se puede concluir que el extracto con el mejor índice de mortalidad sobre Rhipicephalus (Boophilus) microplus fue con la Phytolacca bogotensis K.

Palabras clave: fitoterapia, guaba, metabolitos, ortiga, soxhlet.

Keywords: guaba, herbal medicine, metabolites, ortiga, soxhlet.

Efectos de la administración oral de Cadmio y Plomo sobre estructuras histológicas de hígado y riñón de carneros (Ovis aries)*

Effects of oral administration of Cadmium and Lead on histological structures of liver and kidney of sheep (Ovis aries)

John J Bustamante Cano ${ }^{1}$, MV, Esp, PhD; Walter I Hernández Arroyave², MV, Esp, MSc; Amanda L Chaparro García ${ }^{3}$, Quim, MSc, PhD; Fabián Jiménez Arango ${ }^{4}$; Zoot, MSc

*Financiado por: Vicerrectoría de Investigaciones, Universidad de Pamplona, Norte de Santander, Colombia. Proyecto de convocatoria interna "El carnero (Ovis aries) como modelo experimental en el estudio de la bioacumulación de metales pesados en tejidos de animales de abasto".
${ }^{1}$ Grupo de Investigación en Ciencias Animales, Universidad de Pamplona, Norte de Santander, Colombia. ${ }^{2}$ Grupo de Investigación PROCA, Instituto Universitario de la Paz, Barrancabermeja, Colombia. ${ }^{3}$ Grupo de Investigación en Recursos Naturales, Departamento de Química, Facultad de Ciencias Básicas, Universidad de Pamplona, Norte de Santander, Colombia.

${ }^{4}$ Universidad de Santander, Colombia.

E-mail:fagraria@unipamplona.edu.co

Introducción: Los efectos deletéreos de metales pesados como el Plomo $(\mathrm{Pb})$ y el Cadmio $(\mathrm{Cd})$ sobre las diferentes especies han sido bien documentados. Así mismo, se ha considerado que la ingesta máxima tolerable para ovinos es de $0,2 \mathrm{mg} / \mathrm{Kg}$ peso de vivo y de $1,7 \mathrm{mg} \mathrm{Cd} / \mathrm{Kg}$ de peso vivo para el $\mathrm{Pb}$ y $\mathrm{Cd}$, respectivamente. Sin embargo, aún quedan muchos aspectos por dilucidar acerca de su toxicidad. Objetivo: Describir los cambios histológicos, a través de microscopía óptica, en hígado y riñón de carneros (Ovis aries) durante el suministro de $\mathrm{Pb}$ y $\mathrm{Cd}$ en agua de bebida, determinando simultáneamente sus concentraciones en cada tejido. Métodos: El trabajo se realizó en la Granja "Villa Marina" de la Universidad de Pamplona (Norte de Santander, Colombia). Se alojaron 10 carneros criollos, machos, adultos, de peso promedio de $30 \mathrm{Kg}$, que fueron distribuidos al azar en dos grupos (A) y (B), de cinco animales. Tres animales del grupo A recibieron dosis individuales de $160 \mathrm{ppm}$ de acetato de $\mathrm{Pb} / \mathrm{d}$, vía oral, a los dos restantes se les suministró agua desionizada. Del grupo B tres carneros recibieron dosis individuales de $150 \mathrm{ppm}$ de cloruro de $\mathrm{Cd} / \mathrm{d}$, vía oral, a los otros dos animales se les suministró agua desionizada. El experimento tuvo una duración de 70 d. Una vez finalizado el suministro de los metales se extrajeron muestras de hígado y riñón para histopatología y medición de los metales por espectrofotometría de absorción atómica. Resultados: Los cambios estructurales en hígado, debido a los efectos $\mathrm{del} \mathrm{Pb}$ consistieron en degeneración vacuolar de hepatocitos. El Cd provocó necrosis y hemorragias subscapulares. Las lesiones en riñón ocasionadas por $\mathrm{Pb}$ correspondieron a glomerulonefritis membrano-proliferativa, mientras que el Cd ocasionó fibrosis y necrosis renal. Conclusión: Se detectaron niveles de $\mathrm{Pb}$ en hígado de hasta 2,5 ppm y de Cd hasta de 3,0 ppm, sin hallarse diferencia estadística significativa ( $\mathrm{p} \geq 0,01)$. En riñón, los niveles de $\mathrm{Pb}$ fueron de 2,5 ppm, en tanto que los de Cd fueron de 3,25 ppm ( $\mathrm{p}<0,01)$.

Palabras clave: glomerulonefritis, hepatocitos, metal pesado, necrosis, ovino, toxicidad.

Keywords: glomerulonephritis, heavy metal, hepatocytes, necrosis, ovine, toxicity.

\section{El ácido hipocloroso como tratamiento alternativo en papilomatosis bovina: Reporte de caso*}

\section{Hypochlorous acid as an alternative treatment for bovine papillomatosis: Case report}

Margarita M Caballero Mejía ${ }^{1}$, Tec Agrop, MV; Víctor M Acero Plazas², MV, MSc(c); Harald M Martin Fertig ${ }^{1}$, Fis, MSc.

*Financiado por: Limpiootec SAS, Medellín, Colombia.

${ }^{1}$ Limpiootec SAS, Medellín, Colombia. ${ }^{2}$ Asociación Nacional de Médicos Veterinarios de Colombia (AMEVEC). E-mail: garitacaba@yahoo.com 
Introducción: El ácido hipocloroso es una sustancia natural producida por los macrófagos, tiene una función regeneradora de tejidos y potente efecto cicatrizante. Para que el ácido hipocloroso sea estable en soluciones tópicas debe mantenerse en agua super-electrolizada, la cual favorece la estabilidad del producto y produce la regeneración del tejido al activar el proceso de cicatrización. Objetivo: Describir el efecto del ácido hipocloroso como tratamiento alternativo en casos de papilomatosis en bovinos. Métodos: Se tomaron dos casos clínicos de un predio en el Municipio de San Pedro de Los Milagros, (Antioquia, Colombia), correspondientes a dos novillas de raza Holstein de 22 y 25 meses de edad, presentando extensas lesiones dérmicas en ubre, compatibles con papilomatosis. Ambas hembras recibieron previamente tratamiento convencional contra la papilomatosis, como histovacuna, hemoterapia, diaceturato de diazoaminodibenzamidina más cobre subcutáneo por 45 d. Ninguna de las novillas mostró resolución de la enfermedad, por lo que se procede a realizar un protocolo de aspersión de agua super-electrolizada con ácido hipocloroso a $450 \mathrm{ppm}$ una vez al día en toda la extensión de la lesión durante 5 meses. Semanalmente se evaluaron macroscópicamente midiendo el tamaño de los papilomas y la extensión de los mismos en las áreas afectadas de cada animal, encontrándose un proceso de recuperación y disminución de las úlceras a nivel de los pezones de manera progresiva. Resultados: A los 5 meses se examinan las ubres de los animales, encontrándose casi la resolución de las lesiones de manera completa, sin evidenciar úlceras o efectos secundarios tales como fiebre, anorexia, lesiones compatibles en otras zonas del cuerpo y recidivas. Conclusión: Se evidenció la recuperación de ambos animales, sugiriendo el ácido hipocloroso como coadyuvante en casos compatibles con papilomatosis bovina al ser de fácil aplicación, sin efectos adversos en la presente descripción.

Palabras clave: cicatrización, papilomavirus bovino, verruga.

Keywords: bovine papillomavirus, cicatrization, wart.

Estudio de utilización de medicamentos veterinarios en hatos de las principales zonas de explotación lechera de Cundinamarca (Colombia)

\section{Study of veterinary drugs use in dairy herds of the main productive zones in Cundinamarca (Colombia) \\ Edisson Y Pulido Delgado, MV, MSc; José J López, QF, MSc, PhD(c). \\ Red para el Uso Adecuado de Medicamentos, Universidad Nacional de Colombia, sede Bogotá, Colombia. \\ E-mail:edisony85@hotmail.com}

Introducción: La utilización de medicamentos veterinarios puede presentar prácticas inadecuadas por parte de los médicos veterinarios, médicos veterinarios zootecnistas y personal encargado del manejo del hato, convirtiéndose en un factor importante en la determinación de la calidad de la leche como alimento inocuo. Objetivo: Evaluar el uso de medicamentos veterinarios utilizados en hatos de producción lechera de Cundinamarca (Colombia). Métodos: Se realizó un estudio observacional descriptivo de corte transversal con recolección prospectiva de la información mediante formularios diligenciados con el personal encargado del manejo sanitario de los hatos lecheros en un total de 30 fincas como muestra del estudio. Se evaluaron y caracterizaron las prácticas para el buen uso de medicamentos veterinarios. Resultados: Los antimicrobianos son la clase de medicamentos más utilizados con un $60,7 \%$, el $73 \%$ de las personas involucradas en el uso de los medicamentos no cuentan con el nivel técnico para esta actividad de acuerdo a los lineamientos de buenas prácticas de uso de medicamentos ICA, y el 81,27\% de los medicamentos están compuestos por principios activos que tienen tiempo de retiro y de estos se identificaron 10 principios activos que no deben administrarse a animales cuya leche es destinada al consumo humano. En el $71 \%$ de los hatos destinan la leche de las vacas en tratamiento como alimento para terneraje y mascotas de las fincas, cerca del $50 \%$ de las fincas los cadáveres de las vacas fueron comercializados y en proporciones importantes las diferentes clases de medicamentos eran obtenidos sin fórmula médica. Conclusión: El presente Estudio De Utilización De Medicamentos
Veterinarios (EUMV) debe ser conocido por las instituciones oficiales competentes en la regulación del uso de medicamentos veterinarios, para lograr medidas que disminuyan los factores de riesgo del uso inadecuado y las malas prácticas ganaderas evidenciadas.

Palabras clave: bovinos, hato lechero, medicamento veterinario.

Keywords: bovines, dairy herd, veterinary drug.

\section{Evaluación del efecto de una L-aminoácido oxidasa obtenida del veneno de Bothrops asper de Colombia sobre bacterias causantes de mastitis bovina*}

Evaluation of the effect of an L-aminoacid oxidase from Bothrops asper venom on bovine mastitis microorganisms

Daniel A Castaño Vásquez, Est MV; Sebastián Mejía Builes, Est MV; Silvia Posada Arias, MV, MSc, PhD(c); Juliana Mira Hernández, MV, MSc.

*Financiado por: Corporación Universitaria Lasallista, Caldas, Colombia. Corporación Universitaria Lasallista, Caldas, Colombia. E-mail: jumira@lasallistadocentes.edu.co

Introducción: Una de las principales causas de mastitis bovina es la infección por microorganismos, para cuyo control tradicionalmente se emplean antimicrobianos. La residualidad antibiótica en los productos de origen animal tiene implicaciones económicas por interferir en la producción de lácteos como yogurt y quesos, y son un problema de salud pública al afectar el equilibrio fisiológico de la flora intestinal humana e inducir el desarrollo de resistencia. Se ha encontrado en venenos de serpientes un alto número de sustancias con actividades agonistas o antagonistas sobre proteínas y receptores específicos, convirtiéndolas en fuentes potenciales de diseño de nuevas moléculas con actividad farmacológica. Las L-Aminoácido Oxidasas (LAAOs) son encargadas de proporcionar amonio como fuente principal de nitrógeno en algunos organismos, mientras que en otros contribuye a la defensa contra bacterias (efecto asociado a la producción de peróxido de hidrógeno). Objetivo: Evaluar el efecto de una LAAO proveniente del veneno de Bothrops asper de Colombia sobre los microorganismos causantes de mastitis en hatos lecheros. Métodos: Se realizó el aislamiento de una LAAO mediante cromatografía de baja presión. Se realizó la toma de muestras de leche en hatos lecheros del norte y del oriente antioqueño. El aislamiento de bacterias a partir de las muestras de leche se realizó en el laboratorio de microbiología de Unilasallista y los ensayos de inhibición se realizaron en el laboratorio de ofidismo de la Universidad de Antioquia (Colombia). Resultados: Se aislaron Staphylococcus aureus, Staphylococcus coagulasa negativo, Escherichia coli y mesófilos a partir de las muestras de leche. La fracción rica en LAAO inhibió el crecimiento del 100\% de las cepas probadas, con halos de inhibición sin diferencia estadística con respecto al control positivo empleado (cloranfenicol). Conclusión: La LAAO tuvo una capacidad inhibitoria similar al cloranfenicol mostrándose como una posible alternativa al tratamiento de la mastitis.

Palabras clave: bovino, l-aminoácido oxidasa, mastitis, resistencia antibiótica, veneno de serpiente.

Keywords: antibiotic resistance, bovine, l-aminoacid oxidase, mastitis, snake venom.

\section{Metilprednisolona en altas dosis e infusión continua para el trauma medular agudo en un caprino: Reporte de caso*}

\section{Methylprednisolone in high doses and continuous infusion for} acute spinal cord trauma in a caprine: Case report

Andrés F Castro $\mathrm{Mesa}^{l}$, MV; Víctor M Molina Díaz ${ }^{2}, \mathrm{MV}$, MSc; Camilo Jaramillo Morales $^{2}, \mathrm{MVZ}, \mathrm{MSc}$.

*Financiado por: Corporación Universitaria Lasallista, Caldas, Colombia. ${ }^{1}$ Clínica Veterinaria Lasallista, Corporación Universitaria Lasallista, Caldas, Colombia. ${ }^{2}$ Grupo de Investigación GIVET, Corporación Universitaria Lasallista, Caldas, Colombia.

E-mail: cajaramillo@lasallistadocentes.edu.co 
Anamnesis: Una cabra criolla de 14 meses de edad fue derivada a la Clínica Veterinaria Lasallista (Caldas, Colombia) por caída de tren posterior, con historia de trauma previo. Hallazgos clínicos y de laboratorio: En el examen inicial presenta decúbito esternal, dolor al tacto de la región lumbo-sacra y dolor al manipular la cola, pérdida de propiocepción en miembros posteriores, hiporreflexia ciática y arreflexia patelar además de los signos clásicos de dolor. Se realizaron radiografías latero-lateral y ventro-dorsal de la región lumbosacra, encontrándose compresión medular en el espacio lumbosacro por fractura y desplazamiento ventral del techo del sacro. El hemoleucograma mostró un cuadro típico de estrés, así como un incremento en la fosfatasa alcalina y Alanino Aminotransferasa (AST). Abordaje terapéutico: Se instauró un protocolo para trauma medular agudo nunca antes descrito en caprinos, utilizando metilprednisolona. IV. Se inició con $20 \mathrm{mg} / \mathrm{Kg}, 2$ h después $10 \mathrm{mg} / \mathrm{Kg}, 4$ h después $10 \mathrm{mg} / \mathrm{Kg}$, $6 \mathrm{~h}$ después se instauró una infusión continua de $2,5 \mathrm{mg} / \mathrm{Kg} / \mathrm{h}$ durante $12 \mathrm{~h}$ y dexametasona a $0,2 \mathrm{mg} / \mathrm{Kg}$, intravenosa. La dosis fue disminuyendo cada $12 \mathrm{~h}$ por $3 \mathrm{~d}$ más. Adicionalmente, se corrigió el desbalance hidroelectrolítico y se manejó terapia analgésica con ketoprofeno a $2,2 \mathrm{mg} / \mathrm{Kg}$, IV, cada $24 \mathrm{~h}$ durante $6 \mathrm{~d}$, y gabapentina a $15 \mathrm{mg} / \mathrm{Kg}, \mathrm{VO}$, cada $24 \mathrm{~h}$ durante $5 \mathrm{~d}$. Para estabilizar la lesión neuronal se utilizó DMSO $0,5 \mathrm{~g} / \mathrm{Kg}$, IV (solución al $10 \%$ ) cada $24 \mathrm{~h}$ durante $3 \mathrm{~d}$, y tiamina a $3 \mathrm{mg} / \mathrm{Kg}$, IM, cada $24 \mathrm{~h}$ durante $4 \mathrm{~d}$. Conclusión: La evolución fue rápida y favorable, $24 \mathrm{~h}$ después el paciente se sostuvo en cuadripedestación por $3 \mathrm{~min}, 7 \mathrm{~d}$ después el paciente es dado de alta con capacidad de desplazamiento y con indicación de fisioterapia, 1 mes después se realizó visita y el paciente se desplazaba normalmente. No hubo trastornos metabólicos asociados a la terapéutica, sin embargo, se han descrito algunos asociados a los corticoides. Es procedente buscar alternativas terapéuticas para tratar el trauma medular agudo en caprinos y desarrollar investigaciones al respecto.

Palabras clave: corticoide, decúbito, radiografía.

Keywords: corticoid, radiography, recumbency.

\section{Toxicidad in vitro del fosfato de toceranib en linfocitos de sangre periférica y líneas celulares de carcinoma de células transicionales canino*}

\section{In vitro toxicity of toceranib phosphate in peripheral blood lymphocytes and canine transitional cellular carcinoma cell lines}

Claudia M Castillo Moreno ${ }^{1}, \mathrm{MV}_{\text {, MSc; Johanna Bermudez Lopera }}{ }^{2}$, Bact; Rodrigo A Urrego Alvarez ${ }^{1}$, Zoot, MSc, PhD; Andrés Pareja López², Zoot, $\mathrm{MSc}, \mathrm{PhD}$

*Financiado por: Universidad CES, Medellín, Colombia. ${ }^{1}$ Grupo INCA-CES, Facultad de Medicina Veterinaria y Zootecnia, Universidad CES, Medellín, Colombia. ${ }^{2}$ Grupo Biología CES-EIA, Programa de Biología, Universidad CES. Medellín, Colombia. E-mail: apareja@ces.edu.co

Introducción: Las terapias contra el Carcinoma de Células Transicionales (CCT) son poco efectivas debido a su comportamiento biológico y lugar de presentación, lo cual limita los tratamientos quimioterapéuticos y la completa extirpación quirúrgica. Por lo que se requiere evaluar el uso de diferentes terapias, como los inhibidores de tirosinas quinasas, ya que hay fuerte evidencia que demuestra la efectividad de fármacos de esta familia sobre células que no expresan los receptores aberrantes. Objetivo: Evaluar la actividad citotóxica y genotóxica del fosfato de toceranib en Linfocitos de Sangre Periférica (LSP) y en líneas celulares K9TCC-PU-Original, K9TCC- PU-AxC y K9TCCPU-In. Métodos: Los LSP y las CCT fueron cultivadas con diferentes concentraciones de fosfato de toceranib $(50,100$ y 150$)$ durante $48 \mathrm{~h}$, se evaluó la citotoxicidad en LSP mediante tinción con azul de tripano y en CCT mediante MTT. La genotoxicidad fue evaluada mediante la técnica ensayo cometa. Resultados: El tratamiento con fosfato de toceranib no reduce la viabilidad de LSP ni de las líneas K9TCC-PU-Original y K9TCC- PU-AxC, pero en la línea K9TCC-PU-In, hubo una reducción de la viabilidad relativa (VR) de alrededor del $20 \%$ en las diferentes concentraciones evaluadas. Se encontró genotoxicidad relacionada con la concentración de fosfato de toceranib en LSP, siendo mayor a la dosis más baja. En la línea celular K9TCC-PU-Original y K9TCC-PU-AxC no se encontró significancia $(\mathrm{p}>0,05)$ entre los diferentes tratamientos, pero se evidenció un ligero incremento en la genotoxicidad al incrementar la concentración de fosfato de toceranib ${ }^{\mathrm{en}}$ ambas líneas celulares. La línea K9TCC-PU-In evidenció un claro incremento en la genotoxicidad al incrementar la concentración del medicamento $(\mathrm{p}<0,05)$. Conclusión: Bajo las condiciones experimentales del presente estudio no se evidenció citotoxicidad en LSP ni en CCT. No obstante, se evidenció genotoxicidad del fosfato de toceranib, tanto en LSP como en las CCT, siendo más evidente en K9TCC-PU-In.

Palabras clave: anti-neoplásico, cáncer de vejiga. citotoxicidad, genotoxicidad, líneas celulares, perro.

Keywords: anti-neoplastic, bladder cancer, cellular lines, cytotoxicity, dog, genotoxicity.

\section{Toxicología de la pastura Brachiaria spp en los Llanos Orientales de Colombia}

\author{
Toxicology of Brachiaria spp grasses in Colombian Eastern \\ plains \\ María C Lozano Álvarez, MV, MSc, PhD(c); Gonzalo J Díaz González, \\ $\mathrm{MV}, \mathrm{MSc}, \mathrm{PhD}$. \\ Universidad Nacional de Colombia, sede Bogotá, Colombia. \\ E-mail: mclozanoa@unal.edu.co
}

Introducción: Brachiaria spp es una gramínea común en los Llanos Orientales de Colombia que puede generar en los animales falla hepática (por saponinas o esporodesmina, provenientes del hongo Pithomyces chartarum), hipocalcemia por oxalatos y alteraciones asociadas a la presencia de nitrato. Objetivo: Detectar y cuantificar saponinas, esporas de $P$. chartarum, oxalato y nitrato en Brachiaria spp de la Orinoquía colombiana y relacionar sus niveles con factores climáticos, especie y edad de la gramínea. Métodos: Las herbáceas $B$. brizantha, $B$. decumbens, $B$. humidicola, $B$. dictyoneura y $B$. ruziziensis $\times B$. brizantha $\times B$. decumbens (mulato) fueron muestreadas en cuatro épocas climáticas y en dos tiempos post-corte tanto en piedemonte como en la vega del río Ariari. Se cuantificaron las esporas de $P$. chartarum por microscopía óptica, la protodioscina y diosgenina por técnicas cromatrográficas, los oxalatos totales por titulación oxido-reductiva y el nitrato por espectrofotometría. Se aplicó un diseño factorial $5 \times 4 \times 2$ (especie, época y edad) y correlación de Pearson para reconocer los factores influyentes en la concentración de los compuestos. Resultados: El conteo de esporas de P. chartarum no fue relevante. La concentración de los demás compuestos responde a una interacción de factores. La protodioscina superó el nivel mínimo tóxico (1\%) y en el $77 \%$ de las muestras fue mayor en época seca. La diosgenina, parte estructural de la protodioscina y de otras saponinas, se encontró en concentraciones reportadas como tóxicas. Las especies que más acumularon estos compuestos fueron $B$. decumbens, mulato y B. brizantha. Niveles de nitrato $>2000 \mathrm{ppm}$ en el $72 \%$ de las muestras podrían ocasionar alteraciones reproductivas, mientras que los oxalatos pueden estar presentes en más del $60 \%$ de $B$. humidicola y $B$. dictyoneura en niveles $(>0,13 \%)$ considerado causantes de problemas óseos en equinos. Conclusión: En Brachiaria spp establecida en los Llanos Orientales de Colombia el clima y la especie influyen en la producción de compuestos tóxicos para los animales herbívoros.

Palabras clave: diosgenina, nitrato, oxalatos, protodioscina.

Keywords: diosgenin, nitrate, oxalates, protodioscin. 